\title{
Semantic distance effects in naming superordinates*
}

\author{
A. J. SANFORD $\dagger$ and P. H. K. SEYMOUR \\ The University, Mundee DD1 $4 H N$, Scotland
}

\begin{abstract}
The time to select a superordinate label in response to a target word is shown to be a function of the normative likelihood of the target being produced as an exemplar of the superordinate. This extends the scope of semantic distance effects to a new task situation. The effect declines with practice (repetition) and is also reduced by other preceding tasks. Two alternative accounts of the processing underlying selection are discussed.
\end{abstract}

There is now considerable evidence that not all exemplars of a category are accepted as members of that category with equal speed (Wilkins, 1971; Heider, 1973). Wilkins demonstrated that the speed with which an exemplar was accepted depended upon the normative frequency of that exemplar being given as an instance of the category. Thus CHICKEN is less likely to be produced as an instance of BIRD than is ROBIN (Battig \& Montague, 1969), and it is also accepted as a member of the class BIRD more slowly. Wilkins suggested that the conjoint frequency, or frequency of co-occurrence of exemplar with category, determined the reaction time (RT). Heider (1973) suggested that some exemplars are more "representative" of a category than others, and presented data to prove that Ss could judge how typical of a category a particular instance was. For example, ROBIN was judged as being a better representative of BIRD than was CHICKEN. Both frequency of use and the "representativeness" viewpoints can be described for our current purposes under the neutral title of "semantic distance (SD) effects."

YES/NO classification tasks form only one type of behavior in dealing with superordinates. In the present paper, two other types of behavior are examined in an exploratory manner. Both are superordinate naming tasks in which Ss are given an exemplar and asked to name its superordinate. This corresponds closely to the normal operation of simple classificatory behavior. The principal issue examined in this paper is whether there is an SD effect for the operation of selecting a superordinate from a limited ensemble of possible alternatives (superordinate selection task). Brief consideration is given to a further task, superordinate production, in which Ss are presented with a target and asked to articulate the name of the category to which they would normally assign the target. Thus given the target GOOSE, Ss may respond BIRD, FOOD, WATERBIRD, etc., depending upon the way they would "normally" classify the words. It is arguable that the SD effect could result from the selection task and not occur

\footnotetext{
*'This research was supported by S.S.R.C. Grant No. HR /1833 to the authors. The assistance of Barbara Simmons and Lynn Stanley with the collection and analysis of data is gratefully acknowledged.

Theprint requests to A. J. Sanford at the above address.
}

in the production task if the superordinates allowed in the response set of the selection task did not include the "natural" superordinates of the "less representative" instances. For example, if an S would normally reply FOOD to GOOSE but is only allowed to say BIRD, then he might be delayed accordingly through response competition. In Experiment I, superordinate selection and production latencies are examined as a function of the "distance" between exemplar and category as specified by the Battig and Montague norms. Repeated measures will be taken on the same target words to assess the stability of any SD effect which might occur.

\section{EXPERIMENT I}

\section{Method}

Stimuli were selected by finding two exemplars having a large distance (FAR items) and two having a small distance (CLOSE items) from each of four categories. The four categories were CR IME, CLOTH, SPORT, and BIRD. The corresponding CLOSE items were THEFT, RAPE; COTTON, SILK; GOLF, TENNIS; ROBIN, SPARROW; the poor ones were ARSON, FRAUD FELT, SACK; RIDING, FISHING; and GOOSE, DOVE. Distance was assessed on the basis of the Battig and Montague norms. The words were chosen so that CLOSE and FAR items were matched, on average, with respect to word length and frequency (Thorndike-Lorge).

The control and display of stimuli and the recording of , responses was done by a PDP-12 computer with a VR-14 oscilloscope joint display. The target words were presented with the first letter centered in the middle of the screen. Characters were displayed in standard PDP-12 half-size format, and viewing distance was about $1 \mathrm{~m}$. Room lighting was kept dim so that the characters could be read with ease.

The Ss were 16 unpaid volunteer students and research staff from the University of Dundee. They were naive with respect to the aims of the experiment and were concerned only with keeping their RTs to a minimum, as was suggested by the instructions. Fight Ss wes allocated to the selection task and eight to the production task.

The practice items were not drawn from the experimental ensemble. On each trial of the experiment, the $S$ saw the word NEXT centrally on the screen for $1.5 \mathrm{sec}$, followed by a blank gap of $1.5 \mathrm{sec}$, followed by the target word. The word NEXT acted as both warning signal and fixation point. The target word remained on the screen until $S$ responded. Trial rate was under S's control, each trial being initiated by $S$ 's closure of a microswitch. RTs were measured and recorded in centiseconds by the computer.

The complete session entailed the completion of six trial 


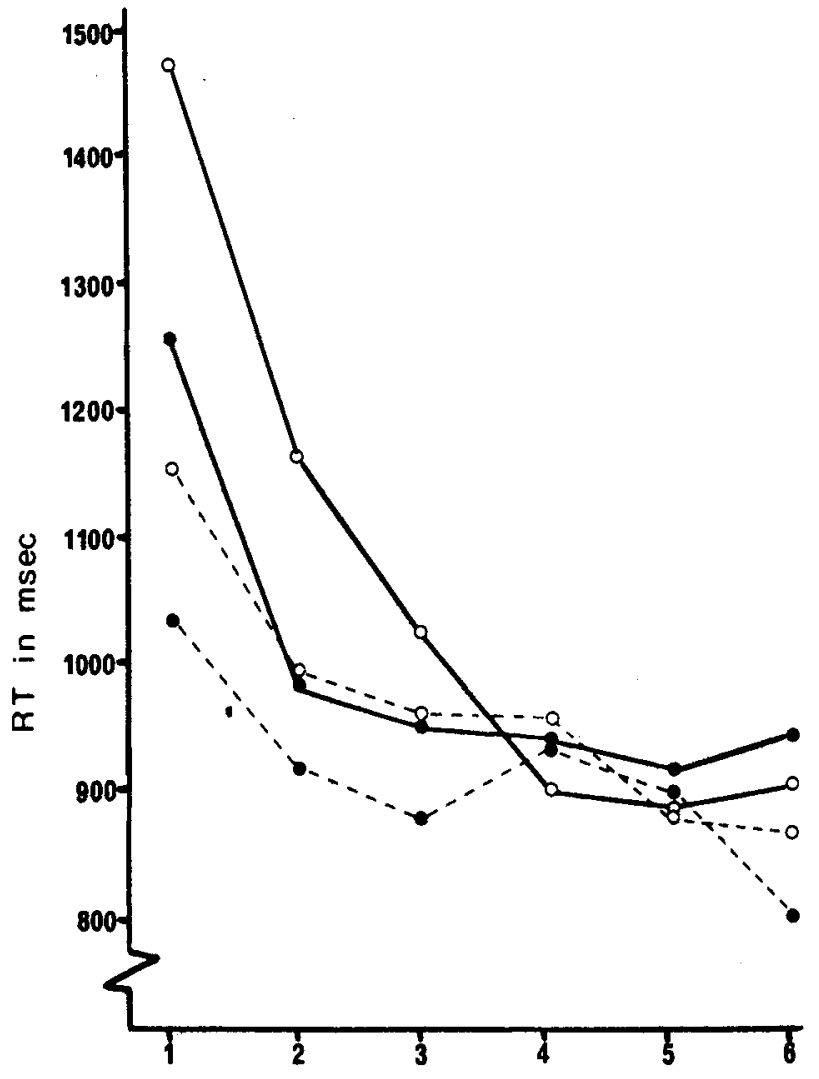

TRIAL BLOCK

Fig. 1. Mean RT as a function of category-item semantic distance and trial block. Open circles: superordinate production; closed circles: superordinate selection. Solid lines: FAR items; broken lines: CLOSE items.

blocks separated by rest instructions. During each trial block, each stimulus was presented once. The order of presentation of stimuli within blocks was random within this constraint and was different for each S. Finally, Ss were instructed to rest when they felt like it and not to initiate a trial unless they were ready to deal with it.

\section{Results}

The results of this and the other experiments in this paper are analyzed by nonparametric statistical tests on untransformed data (Siegel, 1956). First, we will consider the superordinate selection task. In this the error rate was very low, at only $2.7 \%$, most of which was due to failures on the part of the $S$ to activate the voice key. Accordingly, the mean RTs given in Fig. 1 are taken as representative of the RTs associated with almost error-free performance.

The results given in Fig. 1 were obtained by averaging the RTs across all CLOSE and FAR examples in a particular trial block. There was a marked practice effect on overall RT across the six trial blocks $(p<.01$; Friedman test). There was also an overall effect of SD when RTs for CLOSE and FAR items were collapsed across trials ( $p<.02$, Wilcoxon test), RTs to FAR items being greater than those to CLOSE items. The magnitude of the SD effect showed some tendency to decline with trial blocks, and this is represented as a significant effect of trial blocks on the magnitude of the SD effect $(\mathrm{p}<.02$; Friedman test).

Since in later experiments comparison of first trial block exposures are important, it should be noted that the 223-msec first trial block SD effect is significant $(p<.01$; Wilcoxon test). The SD effect is represented in all four categories employed. For CLOTH and SPORT, 8 out of 8 Ss showed the effect; for CRIME, 7 out of 8 ; and for BIRD, 6 out of $8 .^{1}$ This degree of consistency is quite high, since stimuli within semantic categories were not balanced with respect to word frequency and length.

In the case of the production task, a similar pattern of results occurred as shown in Fig. 1; when stimuli were grouped according to the selection task, in 93\% of all cases the superordinate names produced for FAR examples matched those for CLOSE examples. The deviations resulted in the main from difficulties with the word SACK, which was sometimes classified as "CONTAINER" and on one occasion as "WINE." In these cases, it is clearly impossible to make a CLOSE-FAR comparison using these RTs. In other cases, associations of a freer nature occurred. The data presented in Fig. 1 results from the 93\% of cases which were matched in terms of semantic category.

RTs obtained in this way showed a marked effect of practice on overall RTs ( $<<.01$; Friedman test). There was also an overall effect on semantic distance when RTs to CLOSE and FAR exemplars are collapsed across categories and trials $(\mathrm{p}<.05$; Wilcoxon test), RTs to FAR items being greater. The magnitude of this SD effect is a function of trial blocks $(p<.01$; Friedman test), tending to fall off with increasing exposures. Considering the first trial alone, the 318-msec SD effect is significant $(p<.01$; Wilcoxon test), and a breakdown by categories showed six Ss out of eight showing the effect in the expected direction in the categories BIRD, CLOTH, and SPORT and five out of seven showing it in the case of CRIME. The effect was statistically significant $(p<.01)$ in all but the category BIRD. Again, the effect does not seem to be all due to any one of the categories, and the results as a whole mirror those obtained in the selection task. Differences in RTs between the two conditions do not hold up statistically. This applies to both the $95-\mathrm{msec}$ difference between the SD effects on the two tasks in the first trial block and to the difference in mean RT in the two tasks, although Fig. 1 suggests a trend in the direction of greater RTs in the production task. The possibility of a difference between these two tasks may warrant further exploration, however.

\section{Discussion}

The first main effect to emerge from these experiments is the presence of a marked SD effect. The results of the production task suggest that the effect does not result from a preference on the part of the Ss 
for giving different superordinates to CLOSE and FAR items. However, the production task itself does raise some problems. Although Ss were told to produce the superordinate spontaneously, there is the possibility that once they have chosen an appropriate name, e.g., ROBIN $\rightarrow$ BIRD, they may choose to use this name again in a different context, e.g., GOOSE $\rightarrow$ BIRD, when they may otherwise choose a different superordinate. This implies that each $S$ should only have to use one category name once in order to prevent semantic range effects from occurring. Apart from the practical difficulties of measuring SD effects under these conditions, the subtleties of possible contextual priming effects in this type of research are poorly understood, and we suggest that the most profitable procedure would be to attempt to manipulate the semantic range effects directly. In the present case, the semantic range effects can be looked at in two different ways. It may be supposed that Ss deliberately restrict themselves to a limited ensemble of possible responses, from which they make a selection, or that "availability" of some responses becomes so high that they are utilized even under free selection conditions (genuine production). This issue will be raised again in the general discussion, when the mechanisms underlying the selection task are discussed.

The second main effect to emerge is the apparent dependence of the SD effect, as well as mean RT, on the number of exposures to the material. The remaining experiments explore the nature of this reduction in the SD effect, since any reasonable description of the effect should take into account its stability. The next study is an investigation of the effect of exposure to a prior classification task on superordinate selection, and it serves two purposes. Firstly, it enables a check that an SD effect does occur in a YES/NO classification task using the stimulus ensemble of Experiment $I$, and allows a comparison of the relative magnitude of the effect in the two tasks. Secondly, it will show whether use of a specific ISA $^{2}$ relationship will produce a reduction in the latency for using that relationship.

As a preliminary dichotomy, it seems possible that the reduction of the SD effect with practice could be due to either the formation of specific action plans or to the strengthening of the terms in a particular ISA relationship by prior priming. By the specific action-plan idea, we mean that Ss may undergo some sort of memory reorganization as a result of exposure to the task so that seeing a target word, such as GOOSE, results in articulation of the word BIRD. This could be realized by a simple mechanism in which stored with the semantic representation of the word GOOSE is a list of properties (HAS WINGS, ISA BIRD, etc.) and that following exposure to the task, the first "property" to be accessed is a command for action of the type ARTICULATE BIRD. Such a mechanism is clearly very specific and, although effective for the particular task at hand, is of exceedingly limited application. This contrasts markedly with the strengthened ISA notion. In this case, use of the whole proposition structure GOOSE ISA BIRD would effectively alter the semantic distance between the memorial representation of GOOSE and BIRD, regardless of the context in which the proposition structure was used. Thus if an S performs a YES/NO task, in which he decides that GOOSE IS A BIRD, then on a subsequent request for the superordinate for GOOSE, BIRD should be available in less time than if the prior classification task had not been employed. The specific action-plan view would predict no such transfer from classification to superordinate selection. Finally, if tne strengthened ISA view is correct, performance of the superordinate selection task should cause a reduced SD effect in a subsequent YES/NO classification task.

\section{EXPERIMENT II}

\section{Method}

For the classification task, in addition to the 16-category members requiring the answer "YES," 16 unrelated negative items were used. These items were not members of any of the four categories, were not related to them in any obvious way, and were nouns chosen to match the category members in terms of the Thorndike-Lorge word count. All words were presented on the scope of the PDP-12 as in the previous experiments. In the classification situation, rather than being presented with the word NEXT at the start of each trial, a category label from the ensemble of four was presented. Otherwise, the physical parameters governing presentation intervals and position exactly matched those of the previous experiments. Sixteen high school sixth-form students served as Ss, 8 being allocated on a random basis to each group of the design. Group 1 Ss performed the classification task first, then the superordinate selection task, while Group 2 performed the tasks in the reverse order.

Selection task-The instructions given were that the $S$ would see a target word which would be a member of one of four classes which were specified to the $S$. He had to choose the most appropriate class as quickly as possible.

Classification task-The $S$ was told that he would see the category label on the screen and that this would be followed by another word. If the word designated a member of the class, he was to respond YES; if it was not a member, he was to respond NO, either way as rapidly as possible.

Before the $S$ performed either task for the first time, he was given a brief practice session in which only the designated category words were used, but no examples which were to be presented were used. Ss had a brief rest in the middle of the test session, and, as before, trials were self-paced, being initiated by the S's pressing a microswitch. Responses in both tests were verbal, RTs being measured from a voice key closure.

\section{Results}

Mean RTs and error rates for each of the two subgroups in each of the two tasks are shown in Table 1. Since the interpretation of transfer effects is a primary objective of this study, the results will not be treated in the conventional way by averaging conditions across orders. Rather, the first task of the two subgroups will be considered separately, as would be the case in an independent groups design.

Considering performance on the first task, there was a significant SD effect in both the selection situation 
Table 1

Mean Reaction Times for Selection (S) and Classification (CL)

\begin{tabular}{l|lrrrr}
\hline & & Close & Far & SD & Neg \\
\hline Group I & Selection (1st) & 1049 & 1217 & 168 & - \\
S-CL & Classification (2nd) & 717 & 781 & 64 & 910 \\
Group II & Classification (1st) & 806 & 926 & 120 & 973 \\
CL-S & Selection (2nd) & 934 & 991 & 57 & - \\
\hline
\end{tabular}

$(p<.01$; Wilcoxon test $)$ and in the classification situation $(\mathrm{p}<.005$; Wilcoxon), confirming the results of Experiment I and showing that the finding does indeed hold for classification. The SD effect difference between the two tasks, although $48 \mathrm{msec}$, was not significant ( $>$ > 2; Mann-Whitney U test). However, the mean RT was greater in the selection task than in classification $(\mathrm{p}<.01$; Mann-Whitney U test). Finally, in the classification test, negative RTs were signific antly longer than the slowest positive RTs (i.e., those to FAR items) $(p<.01$; Wilcoxon test). We conclude, therefore, that the SD effect is present in the classification task and is of a similar order of magnitude to the SD effect in selection, within the limits of the present design.

It is apparent from Table 2 that when classification precedes selection, the SD effect is substantially reduced. Comparison of the SD effect in the selection situations for Groups 1 and 2 gives a significant difference $(p<.032$; Mann-Whitney test $)$; that is, the $63-\mathrm{msec}$ reduction in the SD effect resulting from prior exposure to the classification situation is significant. Following classification, the SD effect in selection is not significant. Similar comparisons made for the classification task show that the reduction of $104 \mathrm{msec}$ due to prior exposure to the selection situation is significant ( $\mathrm{p}<.005$; Mann-Whitney test). In this case, the reduced SD effect is still significant, however, with $\mathrm{p}<.035$.

\section{Discussion}

The presence of an SD effect in the classification situation gives an independent validation of the difference between the CLOSE and FAR stimuli, as is required by our comments in the introduction. There is also evidence that the reduction of the SD effect from repeated trials in Experiment $I$ can also be obtained by previously exposing the $S$ to a situation in which he has to use the ISA relationship between a specific exemplar-superordinate pair. Thus, priming of the associative connection between the word pairs is sufficient to induce a reduced SD effect, and the production of a specific-action program is not the only consequence of using an ISA relationship in a particular experimental situation. This is confirmed by the fact that the SD effect in classification following a prior selection task is reduced.

When a classification task is encountered by the $S$, not only does he have to utilize the exemplar-superordinate relationship, but he also has experience of reading both the exemplars (16 instances, once) or the superordinate labels ( 4 instances, 4 times). Similarly, on being exposed to the superordinate selection task, the $S$ has experience of reading the exemplars or vocalizing the superordinate labels. It is possible that some reduction in the SD effect could result from reading the exemplars or the superordinate labels.

In the case of the exemplars, an initial exposure to them may result in an increase in the case of reading them. The pertinence of this to the SD effect has been demonstrated in a different context by Meyer, Schvaneveldt, and Ruddy (1972). A lexical decision task was used in which Ss had to decide whether two strings of letters were both words. If they were, and were related associatively, the time to decide YES was less than if they were both words but were unrelated. Thus an association effect could be measured. It was shown that a slight decrease in the ease of reading the stimuli (discriminability) resulted in an increased association effect. Since another theoretical model we will discuss (Morton, 1970) also predicts interactions between association effects and discriminability in some situations, this possibility should clearly be considered here. The Ss taking part in both studies had no particular difficulties in reading the point display of the characters, but there is some possibility that the relatively unusual nature of the characters may have caused some effective reduction in discriminability and that this might be attenuated considerably after the first exposure to the stimuli. Thus the initial large SD effect may result from stimulus discrimination factors. Group 1 of the study which follows read each word once before performing the selection task.

A further possibility is that familiarity with the response set (superordinate names), which obviously increases with trials, might result in an attenuated SD effect. We will reserve discussion of this until after the experiment has been reported, but it should obviously be tested as a possibility, and thus Group 2 in the following study perform a selection task after reading the response set.

\section{EXPERIMENT III}

\section{Method}

The stimuli, apparatus, and temporal and other physical parameters of the display were as specified in Experiment $I$.

Sixteen students from the University of Dundee served as Ss, 8 being allocated to each of two groups on a random basis. All were naive with respect to the aims of the experiment and were

Table 2

Mean Reaction Times in the Stimulus and Response Priming Tasks

\begin{tabular}{lccr}
\hline & Close & Far & SD \\
\hline Unprimed Selection* & 1040 & 1263 & 223 \\
Stimulus Primed & 1023 & 1238 & 215 \\
Response Primed & 1042 & 1124 & 82 \\
\hline
\end{tabular}

*Data from first trial block of Experiment $I$, selection condition. 
concerned solely with keeping their RTs to a minimum.

Ss taking part in the stimulus-primed task were told that they would see some words which they had to read as quickly as possible after they appeared on the oscilloscope display. They received four practice items, followed by the 16 exemplars to be used in the selection task. Following this reading phase, they were given the selection task instructions and performed six trial blocks, each consisting of one presentation of the 16 exemplars. The four practice items used in the reading task were also those used in the selection task and were members of the four superordinate classes serving as responses but were not members used in the main task. In all other respects, the procedure followed that of the selection task in Experiment I.

Ss taking part in the response-primed task were tested in a similar fashion, except that they were presented with each of the four response category words five times each in random order and were instructed to articulate these words when they appeared. In all other respects, the procedure followed that of the stimulus-primed group.

\section{Results}

Mean RTs for the two conditions across six trial blocks following the priming are shown in Fig. 2. Error rates for both tasks were low, being only $2 \%$, all of which was due to failures on the part of the $S$ to activate the voice key cleanly. The stimulus-primed group form an almost perfect replication of the basic results of Experiment 1 , showing a practice effect $(p<.01$; Friedman analysis of variance) and an SD effect $(\mathrm{p}<.01)$. In Table 2, the first trial block RTs are shown for this and other tasks. The SD effect on the first trial block is significant in the stimulus-primed group ( $p<.01$; Wilcoxon test), and it compares closely with the size of the SD effect for the unprimed condition (Experiment I), there being no significant difference between the groups. It can be concluded that stimulus priming does not reduce the SD effect. Accordingly, any explanation of the large SD effect in the first trial block being due to unfamiliarity or difficulty in reading the stimuli must be abandoned.

From Fig. 2 and Table 2 it is evident that there is a dramatic effect of response priming. There is an overall effect of SD, however $(p<.035)$, across , the six trials and on the first trial alone $(p<.035)$, but this is significantly smaller than the SD effect in the unprimed and stimulus-primed situations $(\mathrm{p}<.01$; Mann-Whitney test). It can be concluded that prior experience of reading the superordinate names reduces the SD effect. This, and the other results, can be accommodated into a simple theoretical framework outlined below.

\section{DISCUSSION}

The SD effect appears to be present on first exposure to the stimuli throughout these studies. However, prior exposure to the exemplar-superordinate pairs by repetition on the same naming task or by prior exposure to a YES/NO task reduces the SD effect. This shows that a reduction in SD is not always due to the formation of a specific-action plan or "instruction" to articulate the previously produced response (the superordinate) given

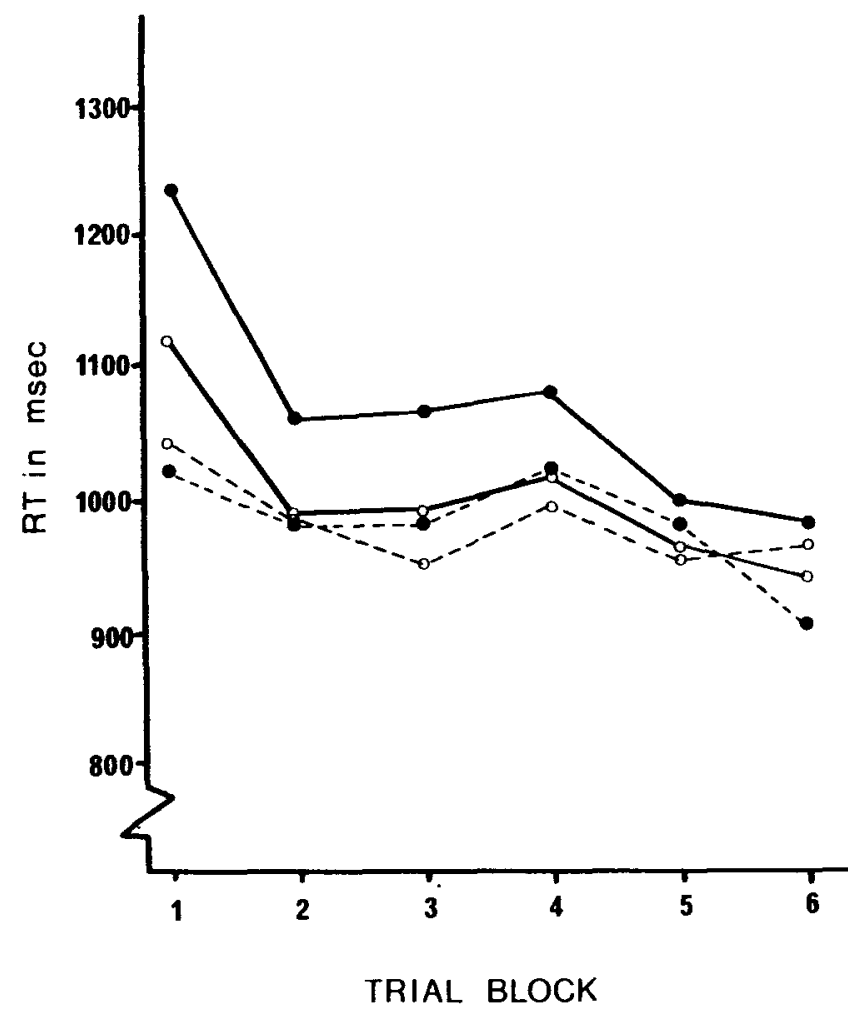

Fig. 2. Mean RT as a function of category-item semantic distance and trial block. Open circles: response-primed group; closed circles: stimulus-primed group. Solid lines: FAR items; broken lines: CLOSE items.

the exemplar. This follows from the fact that vocalization of the superordinate was not required in the YES/NO task and that Ss taking part in that task were not told that they would be asked to name the superordinates in a subsequent task. It is possible, therefore, that FAR items may become closer to the superordinate after they have been used in relation to that category in a prior task. The finding that merely reading the response terms prior to being told that these words could serve as responses in the selection task caused a consequent reduction in the SD effect is less readily explained. One way in which the above findings can be accommodated is explained below and briefly contrasted with alternative formulations.

Morton's (1970) logogen model of word encoding and production offers a suitable framework for the analysis of the selection task. Briefly, a logogen is an accumulator, or counting device. Each word is represented in a lexical dictionary by a logogen, and sensory information that a word has been presented is accumulated by the logogen of that word.

When sufficient evidence has been accumulated, the logogen threshold is exceeded and a symbol, the name of the word, is made available in an output buffer. From here articulation ("naming") can be effected. Excitation of a specific logogen in this way also activates connections in the semantic system between logogens, and logogens for words similar in meaning to the first 
logogen activated receive more activation in this way than do less similar logogens. Thus a logogen can accept inputs from both the sensory and the semantic system; hence the model easily accounts for the interaction between these two sources of evidence (Morton, 1964, 1968). With the additional and plausible assumption that the use of a logogen temporarily lowers the threshold of that logogen, some strong predictions regarding the additivity and interaction of factors in the present experiment might be made.

In a pure production task, the $\mathrm{S}$ has to retrieve a suitable superordinate without having any reference to an experiment-produced response ensemble. The ease with which an $\mathrm{S}$ can produce, say, FURNITURE in response to CHAIR suggests that the superordinate is produced by a rule operating on the target which points to the superordinate, although some exceptions to this may arise (e.g., ANIMAL may be difficult to superordinate, and a superordinate may have to be arrived at deductively). For simplicity it will be assumed that ISA links can be readily identified and thus act as operators in conjunction with a target word.

The SD effect may be handled in two different ways within this framework. It may be supposed that when the threshold of a FAR logogen is exceeded, the time taken for the activation to pass through the semantic system to the superordinate logogen is longer than that for the activation from a CLOSE logogen. Thus the distance effect is explicable in terms of the time for the message to pass from the input to the output logogen. This is in the spirit of a true "distance of separation" account. This contrasts with the view that when a FAR logogen is activated, the quality of activation may be reduced relative to when a CLOSE logogen is activated, i.e., the rate at which activation is accumulated by the superordinate logogen might be less, regardless of the onset latency of the accumulation process. Such a situation would accrue if the ISA link is thought of as comprising a number of functional channels, each of which contributes to the flow of activity into the superordinate logogen. This scheme is in the spirit of "number of common element" or "strength of connection" models.

The effect of restricting the response set can be thought of as lowering the thresholds of the response logogens, causing the appropriate superordinate to come to mind or be "made available" when a compatible target stimulus is displayed. Further, the more rehearsed is the response set, the lower the thresholds become. Now if the rate of delivery of excitation is slower for FAR items, then an interaction between response priming and SD would be expected, since the RTs are defined by the point in time at which the accumulated activation level crosses the logogen threshold. (This is formally equivalent to the explanation of latency differences to signals of different intensities in simple RT, where analogous arguments apply. See Grice, 1968, and Sanford, 1972.) If the SD effect resulted from the time it took a message to pass from the exemplar to the superordinate, only the starting point of the evidence accumulation process would be affected, not its slope, and no interaction between response priming and SD would be expected. The SD effect appears to be mediated by differences in the nature of information transfer rather than by a delay in the transfer as a function of distance. Finally, for consistency it would be expected that the SD effect would be larger in the case of the production task than the selection task (Experiment I) if selection is like production but with reduced superordinate logogen thresholds. It is possible, however, that after a particular superordinate had been used once, its threshold would be reduced to a degree similar to that normally used in the selection task. Thus, although technically Ss could produce a wide range of superordinates in the production task, "restrictions" may occur because of the lowering of certain superordinate logogen thresholds.

An alternative way of viewing the selection task is that Ss search through each of the set of superordinates given, performing an implicit check on whether a target fits an exemplar. Such searches do appear plausible when the $\mathrm{S}$ is set the task of deciding whether a word is a member of one of a number of possible categories (Juola \& Atkinson, 1971; Meyer, 1973). If the $S$ is supposed to search through the members of each category item by item, it is difficult to see how overlearning the categories could result in a reduced SD effect. One possible way out is to suppose that the $S$ simply chooses the superordinate alternative with the greatest semantic similarity to the target. In this case, "similarity" would be accumulated over time and at a faster rate for CLOSE than for FAR examples. Similarity would be accumulated until a criterion level had been passed for one of the possible responses. An interaction might occur between the SD effect and response priming if it is assumed that response priming lowers the criterion level. There is little to choose between this comparison model in which the response produced is the superordinate name most similar to the target and the production system based on the logogen theory, since both are consistent with the observed pattern of results. Both have a certain intuitive plausibility, and the exploratory studies carried out suggest that both must be entertained as possibilities. Of course, a pure production situation, uncontaminated by restrictions on response ensemble (if this is ever really possible), would have to rely on a production scheme of the kind discussed above.

\section{REFERENCES}

Battig, W. F. \& Montague, W. E. Category norms for verbal items in 56 categories: A replication and extension of the Connecticut category norms. Journal of Experimental Psychology Monograph, 1969, 80, 1-46.

Collins, A. M., \& Quillian, M. R. Retrieval time from semantic memory. Journal of Verbal Learning \& Verbal Behavior, $1969,8,240-247$.

Grice, G. R. Stimulus intensity and response evocation. Psychological Review, 1968, 75, 359-373.

Heider, E. R. On the internal structure of perceptual and 
semantic categories. In $T$. M. Moore (Ed.), Cognitive development and acquisition of language. New York: Academic Press, in press.

Juola, J. F., \& Atkinson, R. C. Memory scanning for words versus categories. Journal of Verbal Learning \& Verbal Behavior, 1971, 10, 522-527.

Meyer, D. E. Correlated operations in searching stored semantic categories. Journal of Experimental Psychology, 1973, 99. 124-133.

Meyer, D. E., Schvaneveldt, R. W., \& Ruddy, M. G. Activation of lexical memory. Paper presented to the Psychonomic Society, St. Louis, Missouri, November 1972.

Morton, J. The effects of context on the visual duration thresholds for words. British Journal of Psychology, 1964, 55, 165-180.

Morton, J. Grammar and computation in language behavior. In J. C. Catford (Ed.), Studies in language and language behavior. (Progress Report No. VI) Ann Arbor, Mich: Center for Research in Language and Language Behavior, 1968.

Morton, J. A functional model for memory. In D. A. Norman (Ed.), Models of human memory. New York: Academic Press, 1970 .

Sanford, A. J. Loudness and simple reaction time. Sound, 1972, $6,92-96$
Siegel, S. Nonparametric statistics. New Yoxk: McGraw-Hill, 1956.

Sternberg, S. The discovery of processing stages: Extensions of Donder's method. In W. G. Koster (Ed.), Attention and performance II. Amsterdam: North-Holland, 1969.

wilkins, A. J. Conjoint frequency, category size and categorization time. Journal of Verbal Learning \& Verbal Behavior, 1971, 10, 382-385.

\section{NOTES}

1. This breakdown by category was applied only to the first trial, on which the effects in general were largest. The effect was not significant for the category BIRD, although the trend was strongly in the correct direction.

2. An ISA relation is the directional link connecting an exemplar with a superordinate, e.g., GOOSE-ISA-BIRD.

(Received for publication October 21, 1973; revision received April 15, 1974.) 